\title{
Mesenchymal stem cells regulate inflammatory milieu within degenerative nucleus pulposus cells via p38 MAPK pathway
}

\author{
YUANTING ZHAO $^{1}$, YUE QIN ${ }^{2}$, SHUFANG WU $^{3}$, DAGENG HUANG ${ }^{1}$, \\ HUIMIN HU $^{1}$, XINLIANG ZHANG $^{1}$ and DINGJUN HAO ${ }^{1}$ \\ Departments of ${ }^{1}$ Spine Surgery and ${ }^{2}$ Anesthesiology, Honghui Hospital, Xi'an Jiaotong University, \\ Xi'an, Shaanxi 710054; ${ }^{3}$ Center for Translational Medicine, First Affiliated Hospital \\ of Xi'an Jiaotong University, Xi'an, Shaanxi 710061, P.R. China
}

Received April 2, 2019; Accepted February 5, 2020

DOI: $10.3892 /$ etm. 2020.9150

\begin{abstract}
It has been established that excessive apoptosis of nucleus pulposus cells (NPCs) are responsible for pathogenesis of human intervertebral disc degeneration (IDD). The present study aimed to shed light on the molecular mechanisms underlying the protective effects of mesenchymal stem cells (MSCs) on NPCs in an inflammatory environment. NPCs were treated with TNF- $\alpha$ to induce inflammation and then co-cultured with Wharton's Jelly-derived MSCs (WJ-MSCs) without direct interaction. The levels of inflammation markers (IL-1 $\beta$, IL-6 and IL-8) in NPCs were detected by performing enzyme-linked immunosorbent assay (ELISA), and expression of metalloproteases and aggrecan, as well as the activity of p38 MAPK pathway were determined through immunoblotting. SB-203580 was used to inhibit p38 signaling, prior to evaluation of the effects of Wharton's Jelly-derived MSCs (WJ-MSCs) on inflammatory response within the co-cultured NPCs. After TNF- $\alpha$ treatment, the levels of inflammatory cytokines, MMP-3, and MMP-13 in NPCs were increased whereas aggrecan was decreased, which was then dramatically reversed by WJ-MSCs co-culture. Likewise, WJ-MSCs suppressed TNF- $\alpha$-induced phosphorylation of p38 MAPK signaling components including p38, ASK-1, MKK-3 and MKK-6. Blocking p38 MAPK pathway enhanced the anti-inflammatory impact of WJ-MSCs, and there was no significant difference between NPCs co-cultured with WJ-MSCs or the cells cultured alone. WJ-MSCs co-culture
\end{abstract}

Correspondence to: Dr Yue Qin, Department of Anesthesiology, Honghui Hospital, Xi'an Jiaotong University, 555 Youyi East Road, Beilin, Xi'an, Shaanxi 710054, P.R. China

E-mail: 361827482@qq.com

Dr Dingjun Hao, Department of Spine Surgery, Honghui Hospital, Xi'an Jiaotong University, 555 Youyi East Road, Beilin, Xi'an, Shaanxi 710054, P.R. China

E-mail: doczyt@163.com

Key words: mesenchymal stromal cell, intervertebral disc degeneration, p38/MAPK, anti-inflammation mitigate TNF- $\alpha$-induced inflammatory response and ECM degeneration in NPCs, the major pathological events are implicated in IDD development, probably by suppressing the p38 MAPK signaling cascade.

\section{Introduction}

Low-back pain is a worldwide socio-economic problem that affects more than half the population during their whole life $(1,2)$, and intervertebral disc degeneration (IDD) has been extensively recognized as the most common source of this disorder. Currently, advances in our understanding of the IDD pathogenesis have contributed to the emerging novel therapeutic strategies for retarding the progression or deterioration of it, ranging from pain palliation at early stage to invasive operations at the late stage (3). Unfortunately, these treatment options are not capable of effectively restoring the normal physiological functions of intervertebral disc because of the non-renewable characteristic of nucleus pulposus (NP), one of the major components of intervertebral disc. In addition, surgical interventions for IDD such as spinal arthroplasty and discectomy are usually susceptible to infection and many other complications, hence it is a predominant challenge for spinal surgeons.

IDD is generally characterized by an increase release of pro-inflammatory cytokines, including TNF- $\alpha$ and IL- $1 \beta$, which will recruit the activated immune cells into degenerated tissues and further trigger the inflammatory cascade (4). On the other hand, the inflammatory milieu is also responsible for the aberrant activity of MMPs, ADAMTSs and disintegrins, leading to the interruption of ECM homeostasis $(5,6)$ and thus exacerbating the degenerative process. There are increasing number of studies addressing the utilization of mesenchymal stem cells (MSCs) in the treatment of IDD. Previous studies have proved that MSCs differentiate into NP-like cells with elevated expression of NP-related marker genes when co-cultured with nucleus pulposus cells (NPCs) (7). It was also demonstrated that the MSCs could restore the molecular environments of matrix (8), in addition to its capacities of immunomodulation and multilineage differentiation (9). Hitherto, the mechanisms underlying the effect of MSCs on the degenerative process in NPCs and 
the involved pathophysiological signaling pathways have not been fully described.

Herein, the effect of MSCs on inflammatory response within NPCs was evaluated after exposure to pro-inflammatory cytokine stimulation, and the pathophysiological pathways that may be involved were also investigated. Our results provide insights into the mechanisms underlying the effects of MSCs on TNF- $\alpha$-induced inflammatory response in NPCs, which will further facilitate the application of stem cells in the therapy for the degenerative process.

\section{Materials and methods}

NPCs isolation and culture. The nucleus pulposus samples were collected from patients undergoing surgery from Jan 2016 to May 2018. The magnetic resonance classification of IDD was performed according to a previous report (10). Written informed consents were obtained from patients and counterparts and all the experimental protocols were approved by the Ethic Committee of Honghui Hospital affiliated to Xi'an Jiaotong University (approval no. 2019-12).

The NP tissues were dissected into pieces of $1.0 \mathrm{~mm}^{3}$ and digested with $0.25 \%$ protease at $37^{\circ} \mathrm{C}$ for $1 \mathrm{~h}$, followed by $0.2 \mathrm{mg} / \mathrm{ml}$ of collagenase type II for $4 \mathrm{~h}$, prior to filtration through a sterilized mesh with $70-\mu \mathrm{m}$ pore size and centrifugation at $300 \mathrm{x} \mathrm{g}$, at $4^{\circ} \mathrm{C}$ for $5 \mathrm{~min}$. Cells were resuspended and maintained in DMEM/F12 medium (Gibco; Thermo Fisher Scientific, Inc.) supplemented with $10 \%$ fetal bovine serum (FBS) and 1\% penicillin-streptomycin (Sigma-Aldrich; Merck $\mathrm{KGaA}$ ) at $37^{\circ} \mathrm{C}$ in $5 \% \mathrm{CO}_{2}$. The medium was replaced every 2-3 days and the primary culture was sub-cultured when cells grew to $\sim 80^{\circ} \mathrm{C}$ confluence. NPCs were incubated with $10 \mathrm{ng} / \mathrm{ml}$ TNF- $\alpha$ (Sigma-Aldrich; Merck KGaA) or equal amount of DMEM medium as a negative control for $24 \mathrm{~h}$ before further assessment.

Isolation and culture of Wharton's Jelly-derived MSCs (WJ-MSCs). The fresh umbilical cord was obtained from in a full term neonate, followed by being washed with pre-cold PBS to remove the blood clot. Afterwards, the Wharton's jelly was isolated from umbilical cord and cut into $1 \mathrm{~mm}^{3}$ fragments, which were incubated with type II collagenase $(0.2 \mathrm{mg} / \mathrm{ml}$, Sigma-Aldrich; Merck KGaA) at $37^{\circ} \mathrm{C}$ for $18 \mathrm{~h}$ and then digested using $2.5 \%$ trypsin for $30 \mathrm{~min}$ with agitation. Finally, the cells were fixed with $2 \mathrm{ml} \mathrm{FBS}$ for $2 \mathrm{~h}$ and then maintained in $8 \mathrm{ml}$ of L-DMEM medium (HyClone; GE Healthcare Life Sciences) containing $1 \%$ penicillin-streptomycin, $50 \mu \mathrm{g} / \mathrm{ml} \mathrm{L-ascorbic}$ acid and $2 \mathrm{mM}$ glutamine at $37^{\circ} \mathrm{C}$ in a humidified atmosphere of $5 \% \mathrm{CO}_{2}$. The culture medium was replaced every 2 days. The cells were passaged when the confluence reached $80-90 \%$. WJ-MSCs from passage 3 were used for determination of cell surface antigens. The identification of MSCs is illustrated in Fig. 1 and MSCs differentiate into NP-like cells, with elevated expression of NP-related marker genes, when co-cultured with NPCs.

Enzyme-linked immunosorbent assay (ELISA). The content of released proteins in cell culture supernatant was measured using specific ELISA kits (R\&D Systems). Briefly, the wells of a high protein binding 96-well plate (Corning Costar, Inc.) were coated overnight with $2 \mathrm{ng}$ of monoclonal antibody against IL-1 $\beta$, IL-6 or IL-8. Wells were then washed 4 times with PBS and blocked for at least $1 \mathrm{~h}$ with PBS containing $10 \%$ FBS, followed by the addition of clear cell supernatants and standard dilutions of recombinant human IL-1 $\beta$, IL- 6 or $\mathrm{IL}-8$, prior to $2-\mathrm{h}$ incubation at $37^{\circ} \mathrm{C}$. After washing thoroughly with a buffer containing PBS and $0.05 \%$ Tween-20, each well was incubated with biotinylated antibody for $60 \mathrm{~min}$ and then washed 3 times. After staining with streptavidin-HRP for $30 \mathrm{~min}$, substrate working solution was added to each well and incubated for 15-20 min in the dark. The reaction was then stopped by the addition of $100 \mu \mathrm{l}$ of $2 \mathrm{M} \mathrm{H}_{2} \mathrm{SO}_{4}$, and the $\mathrm{OD}_{450}$ was measured with an ELISA plate reader (Model 550; Bio-Rad Laboratories, Inc.). Each experiment was independently repeated at least three times.

Western blotting. Primary polyclonal antibodies against human p-p38, p38, ASK-1, MKK-3, MKK-6, MMP-3, MMP-13, aggrecan, and actin were purchased from Abcam. Proteins were extracted from NP tissues or cell lysates with RIPA lysis buffer supplemented with protease inhibitors and phosphorylase inhibitor. The protein concentration was measured using the BCA assay (Pierce) following the manufacturer's instructions. The total proteins were resolved by $10 \%$ sodium dodecyl sulfate polyacrylamide gel electrophoresis (SDS-PAGE) before being transferred to polyvinylidene fluoride (PVDF) membrane, which were blocked with $5 \%$ silk milk for at least $1 \mathrm{~h}$ and incubated overnight at $4^{\circ} \mathrm{C}$ with specific primary antibodies (1:1,000 diluted). After rinsing with TBST, the membrane was incubated for $1 \mathrm{~h}$ at room temperature with HRP-conjugated goat anti-rabbit $\operatorname{IgG}$ (1:3,000 diluted). Finally, the labeled proteins were visualized with ECL substrates (EMD Millipore) before quantitative analysis using AlphaView software (ProteinSimple). Each experiment was independently repeated at least three times.

Co-culture of WJ-MSCs and NPCs. For co-culture without direct cell-cell contact, NPCs $\left(1 \times 10^{6}\right.$ cells/well $)$ were seeded on the bottom of transwell 6-well plates, and WJ-MSC cells were placed onto the apical compartment of a 6 -well polyester track-etched cell culture insert with a membrane pore size of $0.4-\mu \mathrm{m}$ (Thermo Fisher Scientific, Inc). Sequentially, cells were grown in DMEM/F12 medium at $37^{\circ} \mathrm{C}$ in a humidified atmosphere of $5 \% \mathrm{CO}_{2}$. NPCs were cultured on common plates as a control and subjected to further analysis.

Statistical analysis. Data are presented as means \pm standard deviation (SD). All in vitro experiments included at least 3 replicates per group. Groups were compared using the two-tailed Student's t-test for parametric data. All statistical analyses were carried out using SPSS 19.0 software (IBM Corp.). $\mathrm{P}<0.05$ was considered to indicate a statistically significant difference.

\section{Results}

WJ-MSCs suppress TNF- $\alpha$-induced inflammation response in $N P C s$. Pro-inflammatory cytokine TNF- $\alpha$ was used to mimic the inflammatory milieu of IDD, and the expression profiles of several inflammation markers in NPCs were evaluated. 

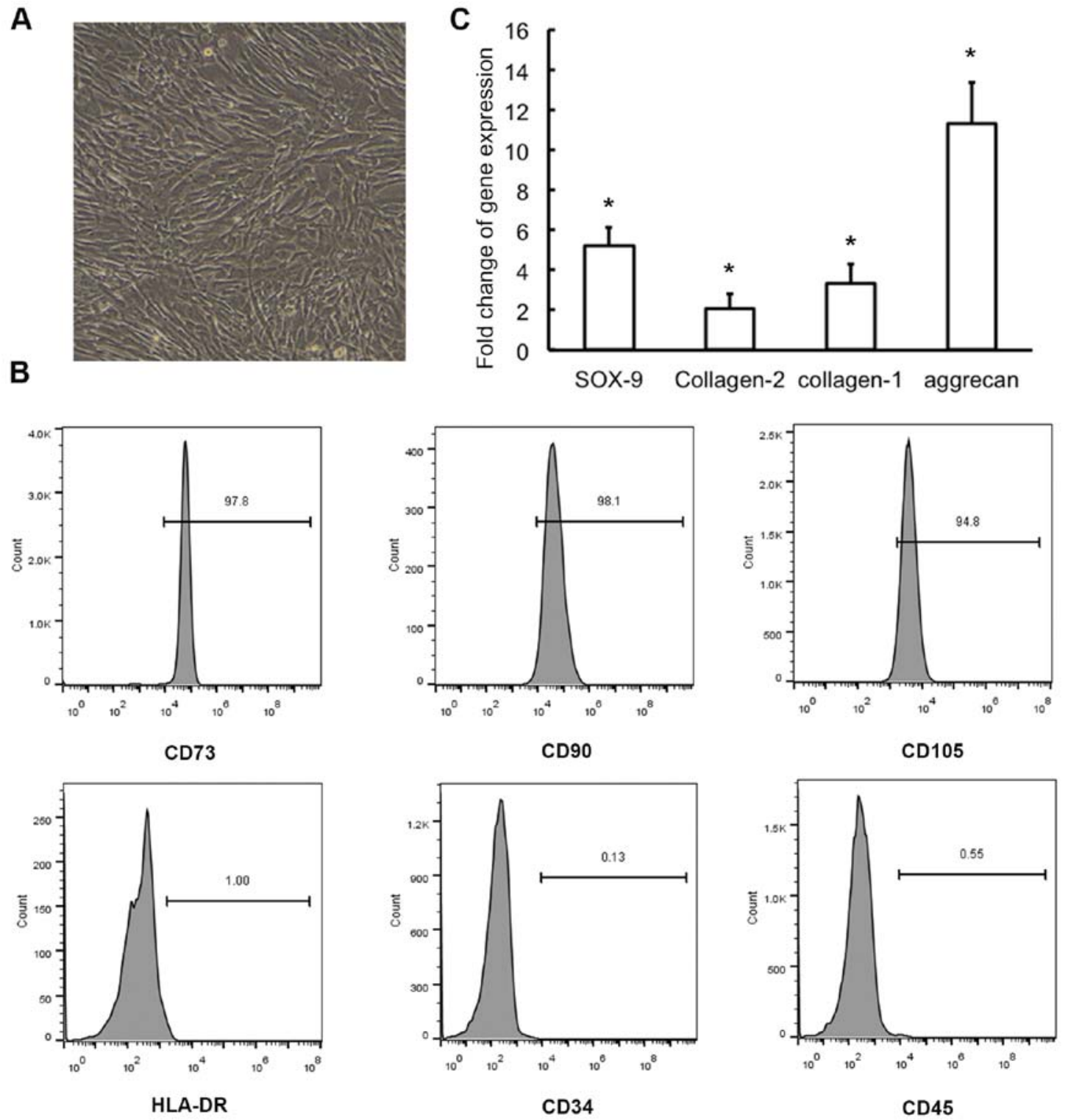

Figure 1. Identification of human WJ-MSCs. (A) Representative image of WJ-MSC spindle-like morphology and adherence to plastic (scale bar, $50 \mu \mathrm{m}$ ). (B) Cell surface markers (CD73, CD90, CD105, CD34, CD45 and HLA-DR) of MSC were tested by flow cytometric analysis. (C) The relative expression levels of gene markers of NP cells were detected in co-cultured WJ-MSCs by qRT-PCR. " $\mathrm{P}<0.05$ compared to gene expression in MSCs cultured alone. WJ-MSCs, Wharton's Jelly-derived MSCs; MSCs, mesenchymal stem cells.

As shown in Fig. 2A, the levels of IL-1 $\beta$, IL-6 and IL-8 were dramatically increased upon TNF- $\alpha$ stimulation. We also observed remarkable augment in the expression of MMP-3 and MMP-13. Inversely, aggrecan level synthesized by NPCs was significantly declined after cytokine treatment (Fig. 2B), suggestive of aggrecan fragmentation and breakdown of hydrated ECM within the nucleus.

TNF- $\alpha$-treated NPCs were co-cultured with WJ-MSCs to determine the effect of WJ-MSCs on the intracellular inflammatory response and the involved signaling transduction. As displayed in Fig. 2A, WJ-MSCs evidently compromised the increased production of IL- $1 \beta$, IL- 6 and IL- 8 by NPCs. In fact, there was no significant difference in the expression of these markers within MSCs, in the presence and absence of TNF- $\alpha$ stimulation (data not shown). Additionally, expression levels of both MMP-3 and MMP-13 were suppressed in NPCs that co-cultured with WJ-MSCs in comparison to cells exposed to
TNF- $\alpha$ alone, whereas aggrecan production was elevated in the presence of WJ-MSCs (Fig. 2B). Therefore, WJ-MSCs can ameliorate inflammatory status within the co-cultured NPCs without direct contact.

WJ-MSCs inhibit p38 MAPK signaling transduction activated by the inflammatory milieu. Following TNF- $\alpha$ stimulation, the phosphorylation of apoptosis signal regulating kinase-1 (ASK-1) was significantly enhanced (Fig. 3). Moreover, the MAPK kinases including MKK-3 and MKK-6 were also activated after incitement by the pro-inflammatory cytokine TNF- $\alpha$, hence promotion of $\mathrm{p} 38$ phosphorylation. The above indicate that the p38 MAPK signaling was activated, which might contribute to the promotion of inflammatory responses during the degenerative process.

Notably, the phosphorylated forms of the above-mentioned molecules implicated in p38 MAPK pathway were found 

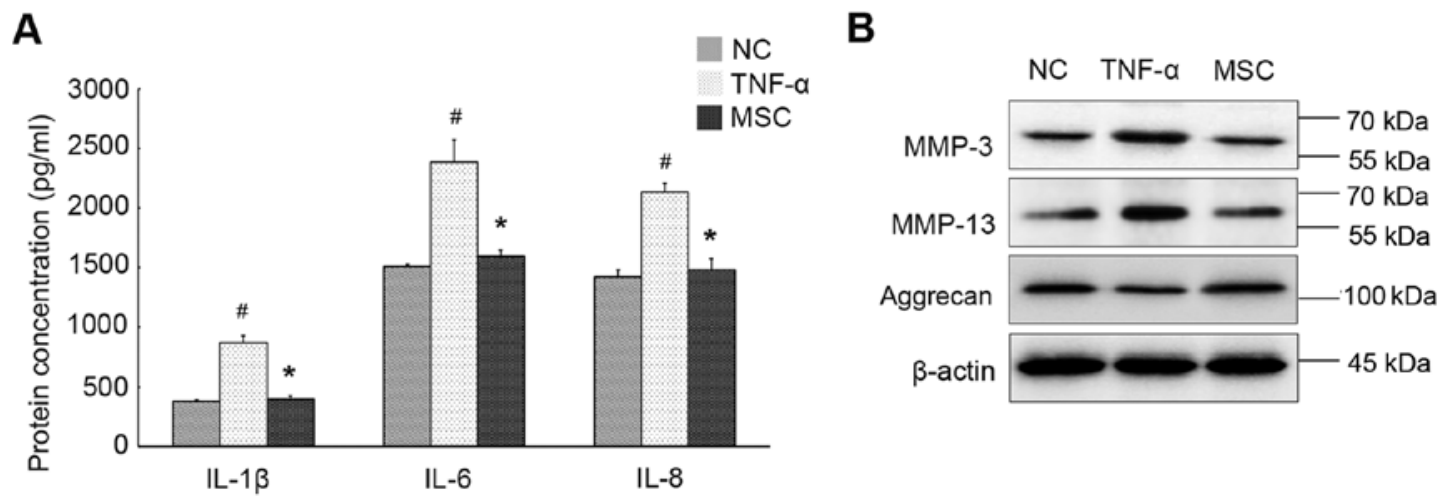

Figure 2. WJ-MSCs suppress TNF- $\alpha$-induced inflammation response in NPCs. (A) ELISA was performed to determine the levels of IL-1 $\beta$, IL-6 and IL- 8 secreted by NPCs that were subjected to different treatments. (B) The protein level of MMP-3, MMP-13, and aggrecan by NPCs was evaluated using western blot assay after different treatments. ${ }^{*} \mathrm{P}<0.05$ compared to the $\mathrm{NC}$ group; ${ }^{\mathrm{P}}<0.05$ compared with the TNF- $\alpha$ stimulation alone. WJ-MSCs, Wharton's Jelly-derived MSCs; MSCs, mesenchymal stem cells; NPCs, nucleus pulposus cells; ELISA, enzyme-linked immunosorbent assay.

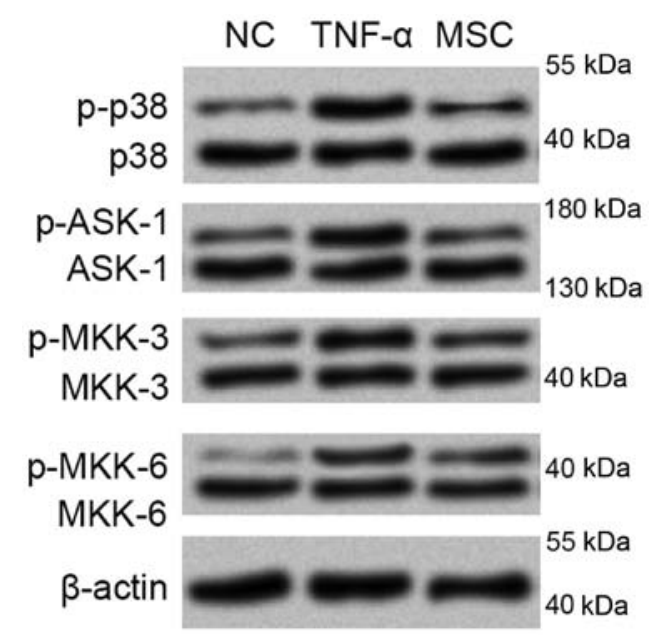

Figure 3. WJ-MSCs inhibit TNF- $\alpha$-induced activation of p38 MAPK signaling. The phosphorylation of ASK-1, p38, MKK-3 and MKK-6 were measured using western blot assay to assess the activities of the corresponding pathways. WJ-MSCs, Wharton's Jelly-derived MSCs; MSCs, mesenchymal stem cells.

decreased due to indirect co-culture between NPCs and WJ-MSCs, suggesting that WJ-MSCs may exert the protective role by virtue of the activation of p38 MAPK pathway.

\section{WJ-MSCs suppress TNF- $\alpha$-inducedinflammation byinhibiting} p38 MAPK signaling. To verify this observation, p38 MAPK signaling inhibitor SB-203580 was utilized and, as expected, it was found that this led to an obvious decrease in the phosphorylation forms of p38 (Fig. 4). Although TNF- $\alpha$-induced MMP-3 and MMP-13 was decreased due to WJ-MSC co-culture, their levels synthesized by NPCs that were co-cultured with WJ-MSCs were further reduced in the presence of SB-203580, whereas aggrecan showed the opposite results. Furthermore, when cells were treated with SB-203580, NPCs co-cultured with WJ-MSCs showed no significant difference from those cultured alone, which suggested that blockage of p38/MAPK pathway protected NPCs against ECM breakdown, regardless of the analogous function of WJ-MSCs. Considering IL-1 1 , IL-6 and IL-8 protection, NPCs exposed to SB-203580 displayed values lower than those observed in the co-cultured cells devoid of SB-203580. Also there was barely detectable difference between NPCs cultured alone and the co-cultured cells when they were subjected to SB-203580 treatment. Thus, we concluded that WJ-MSCs might suppress TNF- $\alpha$-mediated inflammation in NPCs by partially inactivating p38/MAPK signaling.

\section{Discussion}

Not until recently have MSCs been introduced into the therapeutic managements of IDD, emerging as a promising strategy from bench (11-13) to bedside (14). MCSs are probably implicated in the degenerative disc repair in the following aspects: i) complement damaged cells through regeneration of disc-specific cells that have the ability to produce ECM components; ii) control the inflammatory response and (III) promote tissue regeneration by virtue of paracrine signaling factors (15).

Multiple types of tissues are currently proposed as source of MSCs, and among them adipose tissue and bone marrow are predominantly used in IDD-related research. Owing to limited stemness properties and invasive harvesting procedure, MSCs derived from both tissues are restricted in practice (16-19). Herein, WJ-MSCs were introduced in the present study which attempted to provide novel insight into the molecular mechanisms underlying how MSCs play their role in the therapies of IDD disease.

The mitogen-activated protein kinase (MAPK) plays a pivotal role in mediating many cellular processes including responses to inflammation, damage or stress, and cell differentiation. In particular, the implication of p38 MAPK signaling cascades in the pathogenesis of IDD is gradually revealed. By blocking the p38 MAPK pathway, Studer et al (20) found that p38 MAPK was linked to the increased synthesis of inflammation- and pain-related prostaglandin E2 (PGE2) and IL-6, as well as the aggrandized ratio of MMP-3 to TIMP-1. Various stimuli accelerate the breakdown of ECM or inflammation through the activation of p38 MAPK signaling (20-22). In addition, p38 MAPK participate in the regulation of cell senescence of NPCs within the degenerative disc tissue (23). Our results demonstrated that p38 MAPK was initiated upon TNF- $\alpha$ stimulation mimicking the inflammatory microenvironment 
A

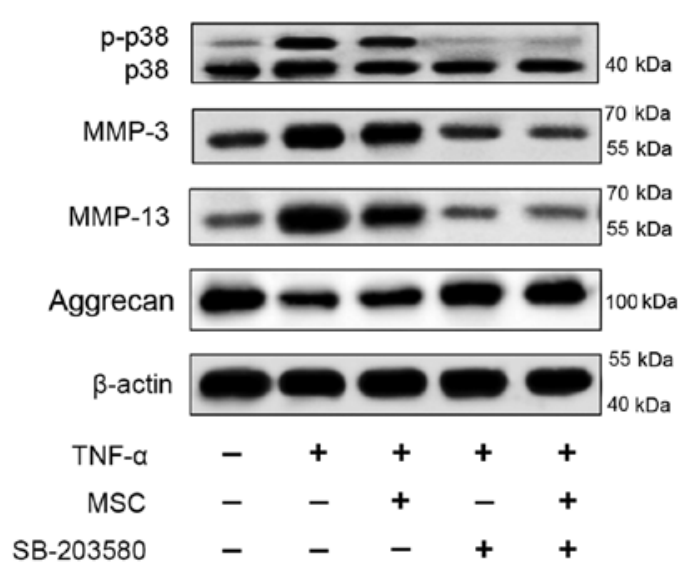

B

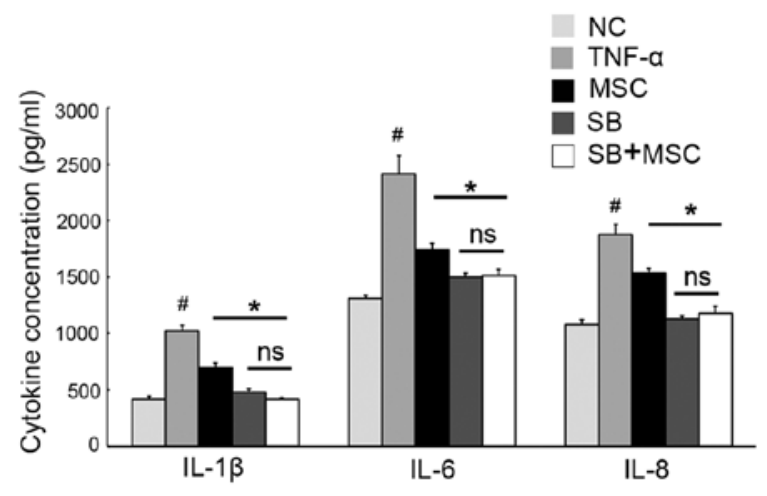

Figure 4. Blocking the p38 MAPK signaling enhances the protective effects of WJ-MSCs. (A) The expression alterations of MMP-3, MMP-13 and aggrecan induced by TNF- $\alpha$ were assessed by immunoblot assay in the presence or absence of SB-203580. (B) The levels of inflammation markers were evaluated via ELISA. ns, not significant; ${ }^{\mathrm{P}}<0.05$ compared to the NC group; ${ }^{*} \mathrm{P}<0.05$. WJ-MSCs, Wharton's Jelly-derived MSCs; MSCs, mesenchymal stem cells; ELISA, enzyme-linked immunosorbent assay.

within IDD, but p38 MAPK and the upstream signaling in NPCs were suppressed when co-cultured with WJ-MSCs. This indicated that WJ-MSCs might exert the effects on inflammatory response through inhibition of p38 MAPK pathway.

Given that MSCs and NPCs were co-cultured without cell-cell interaction, it was suggested that MSCs modulate local inflammatory milieu via paracrine signaling transduction. Afterwards, p38/MAPK inhibitor SB-203580 was utilized and it resulted in an obvious decrease in the TNF- $\alpha$-induced synthesis of inflammatory markers. Considering MMP-3 and MMP-13, NPCs exposed to SB-203580 displayed values lower, whilst a higher amount of aggrecan, than those observed in absence of SB-203580. Inactivation of p38 MAPK pathway can protect NPCs against the detrimental inflammatory response, which is strictly consistent with previous studies. Intriguingly, once cells were pre-treated with SB-203580, levels of all these proteins expressed in NPCs co-cultured with WJ-MSCs were analogous to those NPCs cultured alone, suggesting that blocking p38 MAPK enhanced the protective actions of WJ-MSCs in suppressing inflammatory response. Co-culture with WJ-MSCs might have impact on other signaling pathways that interplay with p38 MAPK during this process. Once p38 MAPK signaling was blocked, there was barely detectable difference between NPCs co-cultured with WJ-MSCs and the cells cultured alone. That is, p38 MAPK signaling plays a predominant role in this process, and WJ-MSCs potentially suppress the TNF- $\alpha$-mediated inflammation within NPCs in a p38 MAPK-dependent manner.

In conclusion, our data demonstrate that after co-culture with WJ-MSCs, TNF- $\alpha$-induced inflammatory response in NPCs was significantly diminished, and the activities of p38 MAPK and the related upstream pathways were decreased, indicating an inhibitory effect of WJ-MSCs on inflammation as well as ECM degeneration by suppressing p38 MAPK activation. Furthermore, blocking p38 MAPK pathway markedly further enhanced the applaudable performance of WJ-MSCs in anti-inflammation, which suggested that WJ-MSCs mitigated the degenerative process within disc tissue by predominantly inactivating p38 MAPK signaling pathway. These findings highlight a possible mechanism underlying the role of MSCs in meliorating the inflammatory milieu within degenerated NPCs, which may be applicable to MSCs-based therapy for disc degeneration.

\section{Acknowledgements}

Not applicable.

\section{Funding}

This study was supported by China Postdcotoral Science Foundation (no. 2016M602943XB), Shaanxi Postdoctoral Research Fund (no. 2017BSHQYXMZZ19), The Fundamental Research Funds for the Central Universities.

\section{Availability of data and materials}

The datasets used and/or analyzed during the present study are available from the corresponding author on reasonable request.

\section{Authors' contributions}

YZ made substantial contributions to the design of the study and the experiments. YQ and SW performed ELISA. DHu and $\mathrm{HH}$ were responsible for immunoblotting. $\mathrm{XZ}$ and $\mathrm{DHa}$ contributed to the analysis of the observation indexes. All authors read and approved the final version of the manuscript.

\section{Ethics approval and consent to participate}

The study was approved by the Ethics Committee of Honghui Hospital, Xi'an Jiaotong University (Xi'an, China). Patients who participated in this research had complete clinical data. Signed informed consents were obtained from the patients and/or the guardians. 


\section{Patient consent for publication}

Not applicable.

\section{Competing interests}

The authors declare that they have no competing interests.

\section{References}

1. McBeth J and Jones K: Epidemiology of chronic musculoskeletal pain. Best Pract Res Clin Rheumatol 21: 403-425, 2007.

2. Mathew J, Singh SB, Garis S and Diwan AD: Backing up the stories: The psychological and social costs of chronic low-back pain. Int J Spine Surg 7: e29-e38, 2013.

3. Zeng Y, Chen C, Liu W, Fu Q, Han Z, Li Y, Feng S, Li X, Qi C, Wu $\mathrm{J}$, et al: Injectable microcryogels reinforced alginate encapsulation of mesenchymal stromal cells for leak-proof delivery and alleviation of canine disc degeneration. Biomaterials 59: 53-65, 2015.

4. Phillips KL, Chiverton N, Michael AL, Cole AA, Breakwell LM, Haddock G, Bunning RA, Cross AK and Le Maitre CL: The cytokine and chemokine expression profile of nucleus pulposus cells: Implications for degeneration and regeneration of the intervertebral disc. Arthritis Res Ther 15: R213, 2013.

5. Risbud MV and Shapiro IM: Role of cytokines in intervertebral disc degeneration: Pain and disc content. Nat Rev Rheumatol 10: 44-56, 2014

6. Loreto C, Musumeci G, Castorina A, Loreto C and Martinez G: Degenerative disc disease of herniated intervertebral discs is associated with extracellular matrix remodeling, vimentin-positive cells and cell death. Ann Anat 193: 156-162, 2011.

7. Yang SH, Yang KC, Chen CW, Huang TC, Sun YH and Hu MH: Comparison of transforming hrowth factor-betal and lovastatin on differentiating mesenchymal stem cells toward nucleus pulposus-like phenotype: An in vitro cell culture study. Asian Spine J 13: 705-712, 2019.

8. Vadalà G, Russo F, Ambrosio L, Loppini M and Denaro V: Stem cells sources for intervertebral disc regeneration. World J Stem Cells 8: 185-201, 2016.

9. Wei A, Shen B, Williams L and Diwan A: Mesenchymal stem cells: Potential application in intervertebral disc regeneration. Transl Pediatr 3: 71-90, 2014.

10. Pfirrmann CW, Metzdorf A, Zanetti M, Hodler J and Boos N: Magnetic resonance classification of lumbar intervertebral disc degeneration. Spine 26: 1873-1878, 2001

11. Yan HS, Hang C, Chen SW, Wang KK and Bo P: Salvianolic acid B combined with mesenchymal stem cells contributes to nucleus pulposus regeneration. Connect Tissue Res: Apr 26, 2019 (Epub ahead of print). doi: 10.1080/03008207.2019.1611794.

12. Cao C, Zou J, Liu X, Shapiro A, Moral M, Luo Z, Shi Q, Liu J, Yang $\mathrm{H}$ and Ebraheim N: Bone marrow mesenchymal stem cells slow intervertebral disc degeneration through the NF- $\kappa \mathrm{B}$ pathway. Spine J 15: 530-538, 2015.
13. Lan WR, Pan S, Li HY, Sun C, Chang X, Lu K, Jiang CQ, Zuo R, Zhou Y and Li CQ: Inhibition of the Notch1 pathway promotes the effects of nucleus pulposus cell-derived exosomes on the differentiation of mesenchymal stem cells into nucleus pulposus-like cells in rats. Stem Cells Int 2019: 8404168, 2019.

14. Arutyunyan I, Elchaninov A, Makarov A and Fatkhudinov T: Umbilical cord as prospective source for mesenchymal stem cell-based therapy. Stem Cells Int 2016: 6901286, 2016.

15. Fierabracci A, Del Fattore A, Luciano R, Muraca M, Teti A and Muraca M: Recent advances in mesenchymal stem cell immunomodulation: The role of microvesicles. Cell Transplant 24: 133-149, 2015.

16. Szychlinska MA, Stoddart MJ, D'Amora U, Ambrosio L Alini M and Musumeci G: Mesenchymal stem cell-based cartilage regeneration approach and cell senescence: Can we manipulate cell aging and function? Tissue Eng Part B Rev 23. 529-539, 2017.

17. Gardner OFW, Musumeci G, Neumann AJ, Eglin D, Archer CW, Alini M and Stoddart MJ: Asymmetrical seeding of MSCs into fibrin-poly(ester-urethane) scaffolds and its effect on mechanically induced chondrogenesis. J Tissue Eng Regen Med 11: 2912-2921, 2017.

18. Szychlinska MA, Castrogiovanni P, Nsir H, Di Rosa M, Guglielmino C, Parenti R, Calabrese G, Pricoco E, Salvatorelli L, Magro G, et al: Engineered cartilage regeneration from adipose tissue derived-mesenchymal stem cells: A morphomolecular study on osteoblast, chondrocyte and apoptosis evaluation. Exp Cell Res 357: 222-235, 2017.

19. Musumeci G, Mobasheri A, Trovato FM, Szychlinska MA, Graziano ACE, Lo Furno D, Avola R, Mangano S, Giuffrida R and Cardile V: Biosynthesis of collagen I, II, RUNX2 and lubricin at different time points of chondrogenic differentiation in a 3D in vitro model of human mesenchymal stem cells derived from adipose tissue. Acta Histochem 116: 1407-1417, 2014.

20. Studer RK, Aboka AM, Gilbertson LG, Georgescu H, Sowa G, Vo N and Kang JD: p38 MAPK inhibition in nucleus pulposus cells: A potential target for treating intervertebral disc degeneration. Spine 32: 2827-2833, 2007.

21. Krupkova O, Sadowska A, Kameda T, Hitzl W, Hausmann ON, Klasen J and Wuertz-Kozak K: p38 MAPK facilitates crosstalk between endoplasmic reticulum stress and IL- 6 release in the intervertebral disc. Front Immunol 9: 1706, 2018.

22. Liu C, Yang H, Gao F, Li X, An Y, Wang J and Jin A: Resistin promotes intervertebral disc degeneration by upregulation of ADAMTS-5 through p38 MAPK signaling pathway. Spine 41: 1414-1420, 2016.

23. Pang L, Li P, Zhang R, Xu Y, Song L and Zhou Q: Role of p38-MAPK pathway in the effects of high-magnitude compression on nucleus pulposus cell senescence in a disc perfusion culture. Biosci Rep 37: BSR20170718, 2017. 